\title{
Understanding transient heat transfer in large-scale ground heat exchanger (GHE) matrices: Insights from high-resolution analytical solutions
}

Min Li

Cheng Zhou

\begin{abstract}
This paper reports on a set of high-resolution analytical solutions, which combines a composite-medium line-source solution, the conventional finite and infinite line-source solutions, and a quasi-3D model for fluid inside U-shaped tubes. This model extends our full-scale line-source model to deal with the thermal interaction between adjacent boreholes and the vertical variation in fluid temperature along $U$-shaped tubes. Based on this model, we analyze both hourly high-frequency temperature responses and long-term temperature evolution of GHEs clusters. The results indicate that 1) the heat capacity of backfilling material can heavily influence the prediction of temperature extrema and 2) the mainstream of heat flux within GHE matrices shifts gradually from the horizontal direction to the vertical direction due to the thermal interaction between GHEs. Finally, this paper suggests a two-borehole approach to approximating the average temperatures of large-scale GHEs matrices.
\end{abstract}

\section{INTRODUCTION}

Inspired by the urgent need for renewable energy, ground-coupled heat pumps (GCHPs) have been globally accepted and increasingly used in large-scale applications. For example, it is not uncommon in China to use GCHPs in buildings having heating and cooling areas greater than 1, $000000 \mathrm{~m}^{2}$. The design and operation of these large-scale systems require detailed knowledge of the heat transfer through ground heat exchangers (GHEs) matrices. A great challenge in the heat-transfer calculations of large-scale GHEs is the diverse time and space scales ( $\mathrm{Li}$ and Lai 2015; Li, et al. 2016). As shown in Li and Lai (2015), four space scales ranging from several centimeters to hundreds of meters and eight time-scales ranging from minutes to decades can be important in the heat transfer of borehole GHEs. A successful model should provide an accurate and efficient approach to calculating the diverse-scale problem. From the perspective of accuracy, the heat transfer analysis should use a model of time resolution ranging from subhour to decades, corresponding to a length range from several centimeters to more than one hundred meters. From the efficient viewpoint, the analysis should tackle the complete spectrum of the time-length scales in an analytical way.

To meet the challenge in modeling GHEs, researchers have developed a vast number of analytical heat transfer models. Among them, conventional finite line-source models (FLS) appear to be the most suitable and efficient Min Li (cnlimin78@gmail.com) is a professor and Cheng Zhou is a MSc student at Central South University. 
models for calculating the long-term temperature response in the ground (Claesson and Javed 2011), but they are unsuitable for modeling the short-term thermal response; the Infinite line-source solution (ILS) is only applicable to calculating the mid-term temperature response (Zeng, et al. 2002); the infinite composite-medium line-source solution (CMLS) emerges as a valid model for calculating the short-term temperature response of GHEs (Li and Lai 2012; Li and Lai 2013; Yang and Li 2014), but it is invalid for the calculation of long-term temperature evolution. An efficient analytical model that can address the entire time-space spectrum will be beneficial. But, very few attempts have been made to develop such a model.

Claesson and Javed (2011) developed a heat transfer model covering time scales from minutes to decades; but they used the equilibrium-diameter assumption for their short-term model, which cannot tackle the complexity inside the borehole in a theoretically complete way (e.g., the position and the number of U-shaped tubes). Li et al. (2014) developed a full-scale line-source model by combining the CMLS and the conventional ILS and FLS solutions using the idea of matched asymptotic expansions. But the solution ignores the thermal interaction between adjacent boreholes and the vertical variation in fluid temperature along U-shaped tubes. Due to the very limited theoretical models, few published studies have adequately explored time-dependent characteristics of large-scale GHE clusters. Without a full understanding of the underground thermal process, geothermal engineers would possess no ability to design appropriate GHEs for the large-scale applications.

The purpose of this paper is, based on our previous work, to help geothermal engineers understand the transient heat transfer in large-scale GHEs matrices. This paper first reports on a high time-resolution analytical solution, a solution that extending the full-scale line-source model to deal with the thermal interaction between adjacent boreholes and the vertical variation in fluid temperature along U-shaped tubes. Next, high-frequency (shorttime) responses and long-term temperature evolution of GHEs clusters are presented. Particularly, this paper is the first attempt to present by heat lines a 3D visualization of heat-flux fields around a large-scale GHEs cluster.

\section{ANALYTICAL MODELS FOR LARGE-SCALE GHE MATRICES}

Heat transfer through GHEs spans a wide range of time and space ranges; thus, to simplify the computation, we decompose the thermal process into two subprocesses ( $\mathrm{Li}$ and Lai 2012; Ma, et al. 2015): 1) the heat transfer from the circulating fluid to the outer wall of the U-pipes (not borehole), which is assumed to be a quasi-steady (or steadyflux) process (Hellstrom 1991; Zeng, et al. 2003), and 2) the heat transfer from the outer U-pipe wall to the ground, which is considered as a transient process. This decomposition considers the heat-storage effect of the heat capacity of backfilling material but ignores those of the circulating fluid and the U-shaped pipes. Suppose that $T_{f 1}$ and $T_{f 2}$ are the fluid temperatures in the legs of the U-pipes (Fig. 1), which are functions of time $t$ and coordinate $z$ in the depth direction ( $z=0$ on the ground surface). The energy balance equations for $T_{f 1}$ and $T_{f 2}$ can be written as (Ma, et al. 2015)

$$
\begin{gathered}
c_{p} m \frac{\partial T_{f 1}(t, z)}{\partial z}=\frac{T_{p 1}(t, z)-T_{f 1}(t, z)}{R_{p}} \\
-c_{p} m \frac{\partial T_{f 2}(t, z)}{\partial z}=\frac{T_{p 2}(t, z)-T_{f 2}(t, z)}{R_{p}}
\end{gathered}
$$

where $R_{p}$ is the fluid-to-pipe thermal resistance (Hellstrom 1991):

$$
R_{p}=\frac{1}{2 \pi k_{p}}\left(\ln \frac{r_{o}}{r_{i}}+\frac{k_{p}}{\alpha r_{i}}\right)
$$

Eqs. (1) and (2) ignore the heat conduction in the fluids along the $\mathrm{z}$-direction because the heat conduction in 
this direction is relatively small, compared with the heat advection of the fluid. These equations consider both the horizontal thermal process through the plastic pipe wall and the vertical variation of the fluid temperature along the U-shaped pipes. The boundary conditions of Eqs. (1) and (2) are the temperature conditions on the ground surface $(z$ $=0$ ) and at the bottom of the borehole $(z=H): T_{f 1}(t, 0)=T_{f, i n}$. $T_{f 2}(t, H)=T_{f 1}(t, H)$, where $T_{f, i n}$ is the inlet temperature of GHEs. $T_{p 1}$ and $T_{p 2}$ in Eqs. (1) and (2) denote the average temperatures on the outer walls of the U-pipe legs (Fig. 1) and generally differ from each other. These temperatures vary linearly with the heat fluxes imposed on the pipe legs; but the average of $T_{p 1}$ and $T_{p 2}$ remains almost unchanged for a given total heat flux (Yang and Li 2014). For example, the average temperature in the case of $50 \%-50 \%$ assignment is identical to the case of $70 \%-30 \%$ assignment for a given total heat flux, because the increased wall temperature due to the increased flux is offset by the decreased wall temperature due to the decreased heat flux. Therefore, it appears to be acceptable to assume that the heat flux is assigned equally to each U-pipe leg, leading to $T_{p 1}=T_{p 2}=T_{a}$. Here $T_{a}$ denotes the average temperature on the outer walls of U-pipe and can be approximated by the arithmetic mean of temperatures of positions $\mathrm{A}$ and $\mathrm{B}$ as labeled in Fig. 1 (Li and Lai 2012). Based on these assumptions and the boundary conditions, the solution to Eqs. (1) and (2) is (Polyanin and Zaitsev 2003)

$$
T_{f, \text { out }}(t)=T_{a}(t)+\left[T_{f, \text { in }}(t)-T_{a}(t)\right] \exp (-2 \eta)
$$

where $\eta$ is defined as $H /\left(c_{p} m R_{p}\right)$, a quantity that have a similar implication as the number of transfer units (NTU) used in the heat exchanger literature. Eq. (4) is applicable only for single U-tube and parallel-connected double U-tubes. The similar idea is applicable to the case of series connected double U-tubes (or W-shaped tubes), and the outlet temperature response is (Ma, et al. 2015)

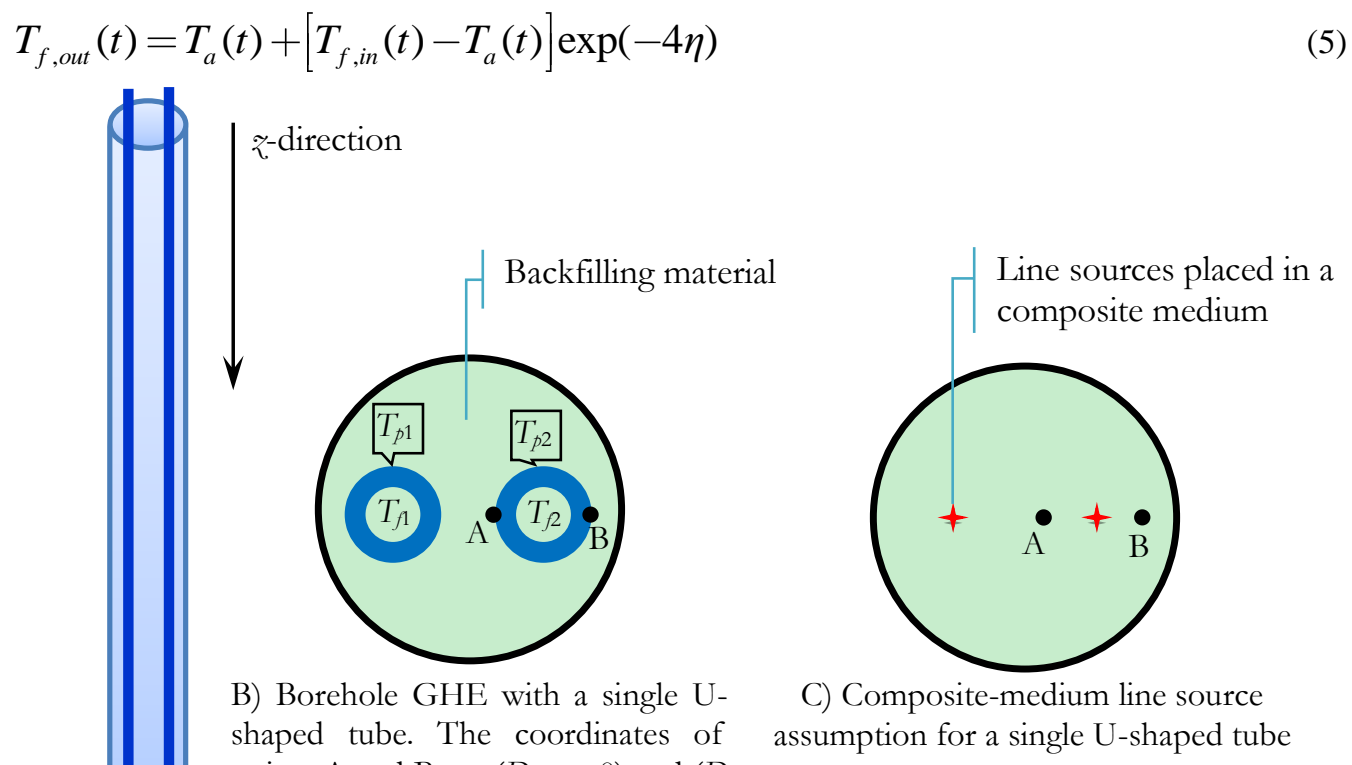
points $\mathrm{A}$ and $\mathrm{B}$ are $\left(D-r_{0}, 0\right)$ and $(D$ $\left.+r_{o}, 0\right)$ in the polar coordinate systems, where $D$ is the half spacing between the pipe legs and the borehole center is the origin.

A) Borehole GHE

Figure 1 The line-source assumption and variable definitions used in the heat transfer model for U-shaped GHEs 
The overall average temperature $T_{a}$ in Eqs. (4) and (5) is unknown and needs to be determined. There are several approaches to determining $T_{a}$. The approach used here is by imposing a constant heat-flux boundary condition on the outer walls of U-shaped pipes because the heat flux can be determined using the heating/cooling loads and the COP of heat pumps. By this approach, $T_{a}$ is the time-dependent average temperature on the U-pipe walls due to the given heat flux on the U-pipe walls; it can be determined using the theory of composite-medium line-source and the idea of matched asymptotic expansion as follows (Li, et al. 2014; Li and Lai 2012):

$$
T_{a}=T_{0}+q_{l} G(t)
$$

$G$ is the temperature response on the outer wall of the $\mathrm{U}$-shaped pipes due to a unit step change in the heat flux (i.e., $q_{l}=1$ in Eq. (6)), consisting of four temperature response functions as follows (Li, et al. 2014):

$$
G(t)=G_{i}+G_{o}-G_{m}+\Delta G
$$

Eq. (7) extended the full-scale line-source model by the additional term $\Delta G$. Here, $G_{m}$ is an infinite line-source solution for the temperature on the borehole wall. (Carslaw and Jaeger 1959):

$$
G_{m}(t)=\frac{1}{4 \pi k_{s}} \int_{r_{b}^{2} / 4 a_{s} t}^{\infty} \frac{\exp (-s)}{s} d s=\frac{1}{4 \pi k_{s}} E_{1}\left(\frac{r_{b}^{2}}{4 a_{s} t}\right)
$$

$G_{o}$ in Eq. (7) is a finite line-source solution for the temperature on the borehole wall. According to Claesson and Javed (2011), the expression for the mean temperature of the borehole wall can be

$$
G_{o}(t)=\frac{1}{4 \pi k_{s}} \int_{1 / \sqrt{4 a_{s} t}}^{\infty} \exp \left(-r_{b}^{2} u^{2}\right) \frac{I\left(H u, H_{1} u\right)}{H u^{2}} d u
$$

where $I$ is a special function defined as follows:

$$
I\left(x_{1}, x_{2}\right)=2 \cdot \operatorname{ierf}\left(x_{1}\right)+2 \cdot \operatorname{ierf}\left(x_{1}+2 x_{2}\right)-\operatorname{ierf}\left(2 x_{1}+2 x_{2}\right)-\operatorname{ierf}\left(2 x_{2}\right)
$$

$\operatorname{ierf}(x)$ denotes integral of the error function $\operatorname{erf}(x)$ :

$$
\operatorname{ierf}(x)=\int_{0}^{x} \operatorname{erf}(s) d s=x \cdot \operatorname{erf}(x)-\frac{1}{\sqrt{\pi}}\left[1-\exp \left(-x^{2}\right)\right]
$$

$G_{i}$ is a composite-medium line-source solution to the average temperature on the outer walls of the U-shaped pipes. The key idea of the composite-medium line-source solution is that the legs of U-shaped tubes (not the borehole) are assumed as lines of heat sources placed in a cylindrical composite medium. Thus, it is possible to obtain the transient temperature field inside the borehole and the average temperature $T_{a}$. Readers can find more details about this model in the references ( $\mathrm{Li}$ and Lai 2012; Li and Lai 2013; Yang and Li 2014). For single U-shaped tubes, $G_{i}$ is

$$
G_{i}(t)=\frac{1}{2 \pi k_{\mathrm{b}}} \sum_{i=-\infty}^{+\infty} \int_{0}^{+\infty}\left[1-\exp \left(-u^{2} a_{\mathrm{b}} t\right)\right] \frac{J_{2 i}\left(u r_{A}\right)+J_{2 i}\left(u r_{B}\right)}{2} \frac{J_{2 i}\left(u r^{\prime}\right)(\varphi g-\psi f)}{u\left(\varphi^{2}+\psi^{2}\right)} d u
$$

For double U-shaped GHEs, the expression of $G_{i}$ is 


$$
G_{\mathrm{i}}(t)=\frac{1}{2 \pi k_{\mathrm{b}}} \sum_{i=-\infty}^{+\infty} \int_{0}^{+\infty}\left[1-\exp \left(-u^{2} a_{\mathrm{b}} t\right)\right] \frac{J_{4 i}\left(u r_{A}\right)+J_{4 i}\left(u r_{B}\right)}{2} \frac{J_{4 i}\left(u r^{\prime}\right)(\varphi g-\psi f)}{u\left(\varphi^{2}+\psi^{2}\right)} d u
$$

where $r_{A}$ and $r_{B}$ are the radius coordinates of points $\mathrm{A}$ and B (see Fig. 1); functions $\varphi, \psi, f$, and $g$ are defined as

$$
\left\{\begin{array}{l}
\varphi=a k J_{n}\left(u r_{b}\right) J_{n}^{\prime}\left(a u r_{b}\right)-J_{n}^{\prime}\left(u r_{b}\right) J_{n}\left(a u r_{b}\right) \\
\psi=a k J_{n}\left(u r_{b}\right) Y_{n}^{\prime}\left(a u r_{b}\right)-J_{n}^{\prime}\left(u r_{b}\right) Y_{n}\left(a u r_{b}\right) \\
f=a k Y_{n}\left(u r_{b}\right) J_{n}^{\prime}\left(a u r_{b}\right)-Y_{n}^{\prime}\left(u r_{b}\right) J_{n}\left(a u r_{b}\right) \\
g=a k Y_{n}\left(u r_{b}\right) Y_{n}^{\prime}\left(a u r_{b}\right)-Y_{n}^{\prime}\left(u r_{b}\right) Y_{n}\left(a u r_{b}\right)
\end{array}\right.
$$

where $a$ and $k$ are dimensionless variable $k_{k}=k_{\mathrm{s}} / k_{\mathrm{b}}, a=\left(a_{\mathrm{b}} / a_{\mathrm{s}}\right)^{1 / 2} ; J_{n}$ and $Y_{n}$ are the derivatives of $J_{n}$ and $Y_{n}$; the order $n$ is equal to $2 i$ and $4 i$ in Eqs. (12) and (13), respectively.

$\Delta G$ in Eq. (7) denotes the superimposed temperature response due to the thermal interaction between adjacent boreholes. Since the thermal interaction between boreholes is a mid- and long-term process (Li and Lai 2015), it should be calculated by the long-term solution, i.e., the finite line-source model (Claesson and Javed 2011):

$$
\Delta G(t)=\frac{1}{4 \pi k_{s}} \int_{1 / \sqrt{4 a_{s} t}}^{\infty} \sum_{i=1, i \neq j}^{M} \exp \left(-B_{i j}^{2} u^{2}\right) \frac{I\left(H u, H_{1} u\right)}{H u^{2}} d u \quad(j=1,2 \cdots, M)
$$

Eq. (15) is identical to Eq. (9) except that $r_{b}$ is replaced by the distance between th ith borehole and the jh borehole under consideration, $B_{i j}$. The summation in Eq. (15) means the superposition of all thermal interaction from the adjacent GHEs. Furthermore, the mean temperature of a GHEs cluster can be approximated by the algebraic mean of all the $M$ boreholes:

$$
\Delta G(t)=\frac{1}{4 \pi k_{s} M} \int_{1 / \sqrt{4 a_{s} t}}^{\infty} \sum_{j=1}^{M} \sum_{i=1, i \neq j}^{M} \exp \left(-B_{i j}^{2} u^{2}\right) \frac{I\left(H u, H_{1} u\right)}{H u^{2}} d u
$$

In Eq. (16), the summation according to $j$ is used to calculate the total temperature response of $M$ borehole GHEs.

\section{RESULTS AND DISCUSSION}

\section{High-frequency temperature responses}

The short-time performance of the analytical models has been validated by comparing the theoretical model to a set of reference sandbox data and a numerical model in our previous work (Li and Lai 2013; Yang and Li 2014). Thus, this section only shows hourly high-frequency prediction. Fig. 2 shows the results of a simulation of a GHEs cluster by inputting a set of hypothetical hourly building loads. The temperature responses to the hourly loads were calculated by applying a conventional line-source model and the full-scale line-source model. Although the heat capacity of backfilling materials is small compared with that of the surrounding soil/rock, its influence on short-term and high-frequency responses is surprisingly significant (Fig. 2): 1) the hourly response given by the full-scale model oscillates with an amplitude clearly smaller than that given by the conventional line-source model; 2) the variation of the temperature responses yielded by the conventional model is entirely consistent with that of the hourly loads; but, the responses produced by the full-scale model do not always follow the pattern of the loads. These two discrepancies can be explained by the damping effect of the heat capacity of backfilling materials on the hourly high-frequency temperatures because the conventional model differs from the full-scale model only in that it ignores the influence of the heat capacity of backfilling materials. In fact, our previous study has illustrated that ignoring the heat capacity 
within bores may lead to a discrepancy in short-term thermal resistance ranging from $26 \%$ to $55 \%$ (Li, et al. 2017).

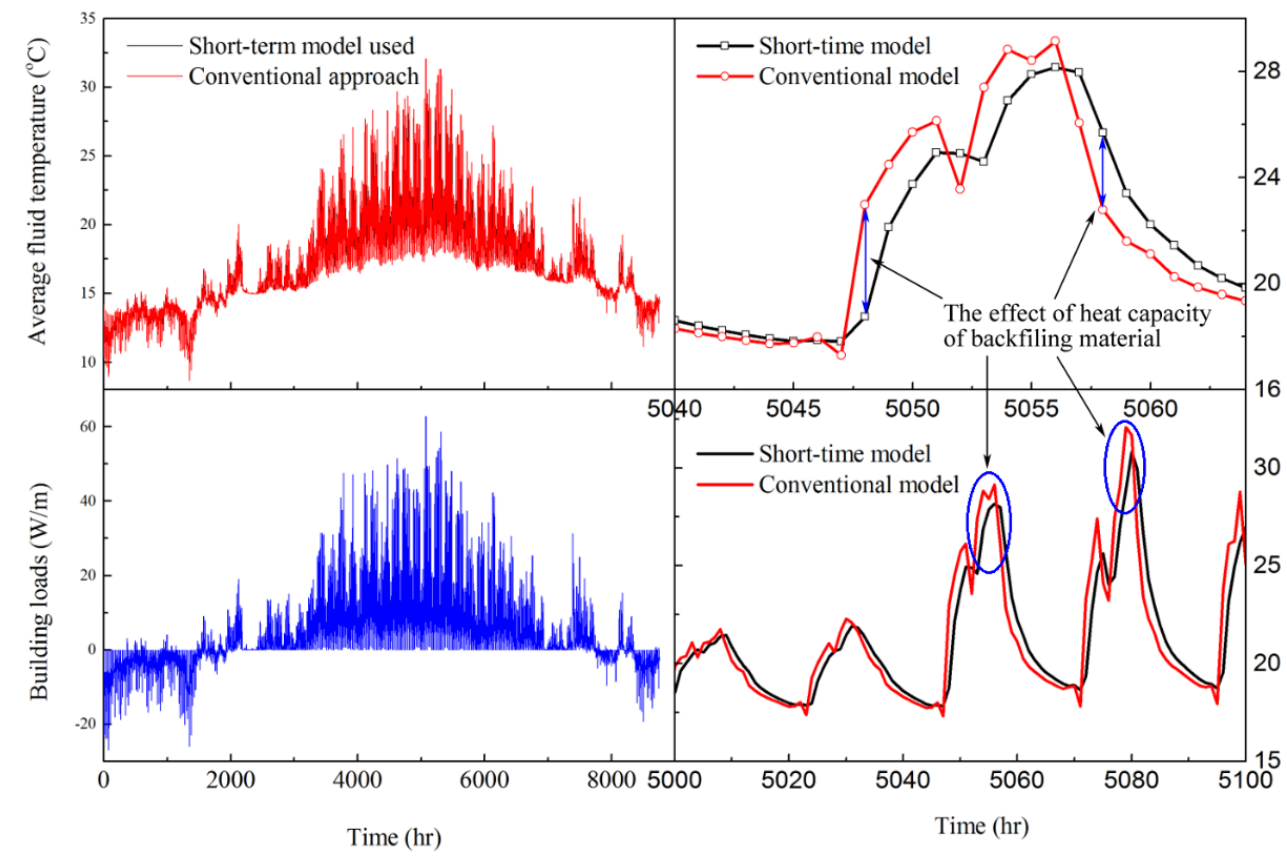

Figure 2 A case study assessing the performance of the full-scale line-source model in predicting high-frequency temperature responses (The conventional model consists of the finite line-source model and a 2-D model for effective borehole thermal resistance)

\section{Long-term underground temperature evolution}

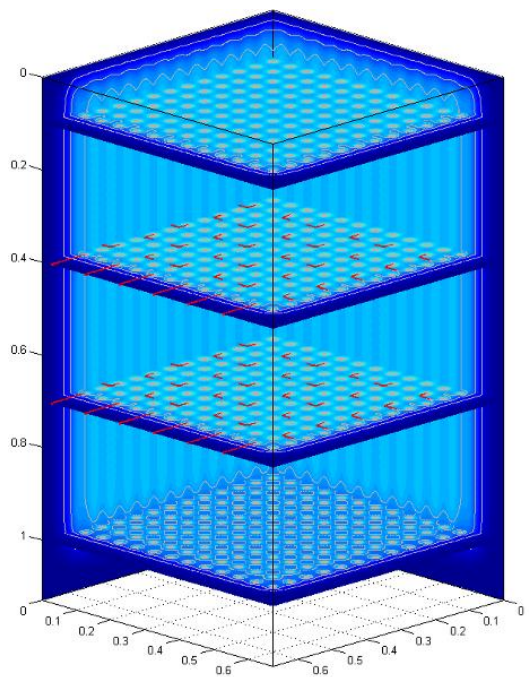

$\mathrm{Fo}=\mathrm{a}_{\mathrm{s}} \mathrm{t} / H^{2}=0.001$

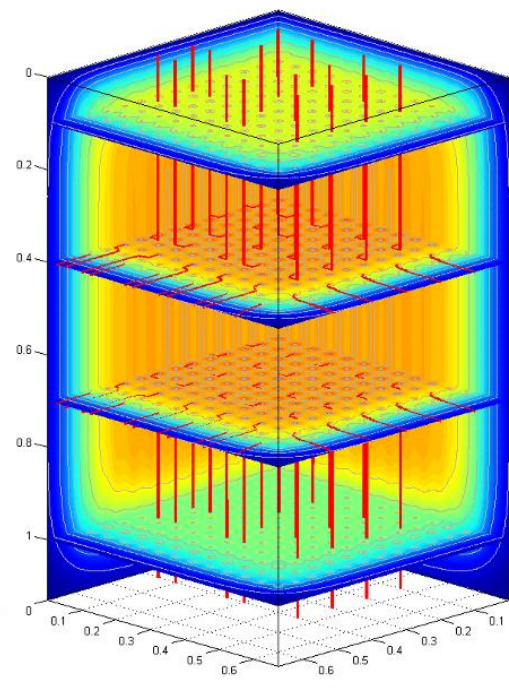

Fo $=0.005$

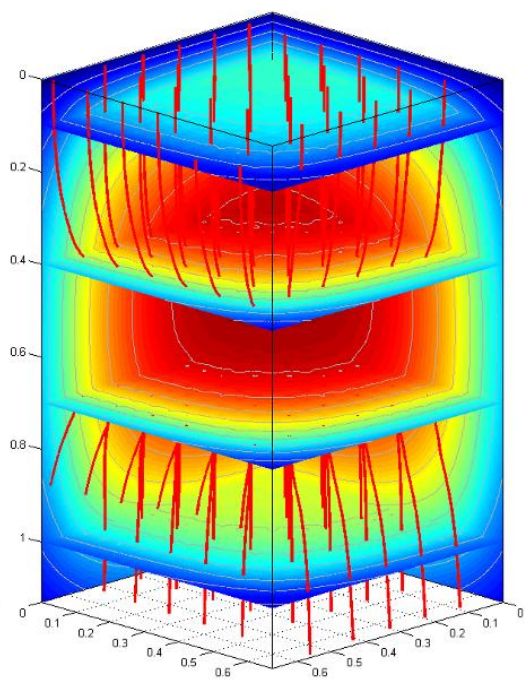

$\mathrm{Fo}=0.05$

Figure 3 3D temperature and heat-flux fields within and around a GHE matrix $(24 \times 24=576)$ at three instants. The red lines represent heatlines that show the direction of heat flux. 
It is necessary for long-term processes to address the thermal interaction between boreholes, which is involved in Eq. (7) using the principle of superposition (i.e., $\Delta G$ ). Fig. 3 shows three snapshots of the 3D underground temperature and heat-flux fields calculated by the new model (576 bores). The heat-flux field is illustrated by the heatlines (the red lines in Fig. 3). A heatline is a vector line whose tangents everywhere coincide with the direction of the heat flux. An interesting observation following from Fig. 3 is that with time increasing the mainstream of the heat fluxes within the GHEs cluster shifts gradually from the horizontal direction to the vertical direction. Especially, the heat fluxes from the inner boreholes become totally vertical (e.g., Fo $=a_{s} t / H^{2}=0.005$ and 0.05 ). The shift in heat-flux directions is caused by the thermal interaction between boreholes and the increase in surrounding ground temperature, which blocks the horizontal transfer of heat. The shift also implies that load imbalance can cause a sharp increase in the mean temperature of large-scale GHE clusters. Moreover, for the purposes of ground heat storage (GHS), improving ground-surface insulation may be a viable means of improving storage efficiency.

\section{Average temperature responses}

For engineering applications, the most important variable should be the average temperature of GHEs clusters, which determines the overall efficiency of GCHP and GHS systems. The analytical model summarized here facilitates heat transfer calculation for large-scale engineering applications. Fig. 4 is one example, which presents a family of $G$ curves for matrix arrangements of bores. While similar charts have been reported by other researchers (Claesson and Javed 2011), Fig. 4 is complementary to previous charts in providing $G$ functions for extremely large clusters and applicable to time scales from minutes to decades.
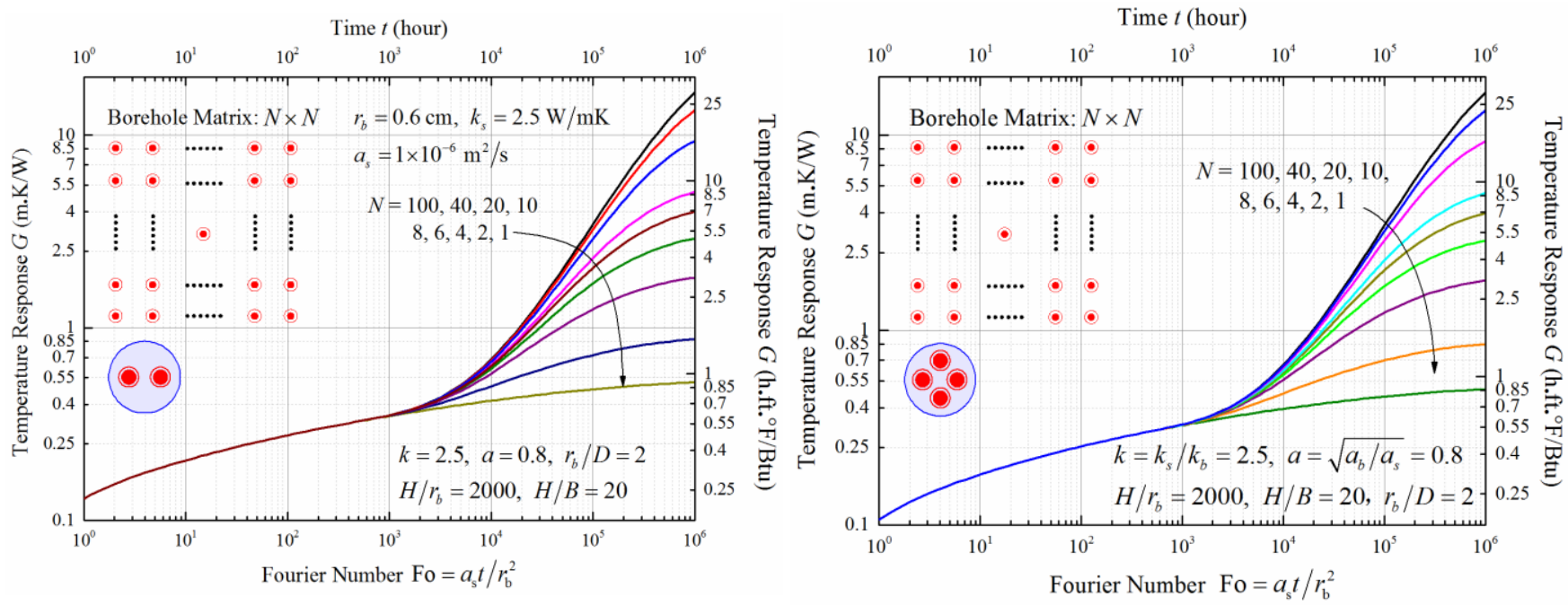

Figure 4 Time-dependent temperature response functions (G-functions) of U-shaped GHEs matrices: From 1 to 10,000 boreholes.

\section{CONCLUSION}

This paper reports on an analytical solutions to heat transfer of GHEs, which can predict temperature responses of GHE matrices from sub-hour to decades. The solution uses the inlet temperature, the flow rate of the fluid, and the initial ground temperature as the boundary/driven conditions for computing the outlet temperature of GHEs, reproducing the physical reality of the operation of GHEs and providing a very direct way of simulation. This feature enables the model to be an idea foundation for analysis, simulation, and design of large-scale GHE clusters. 


\section{NOMENCLATURE}

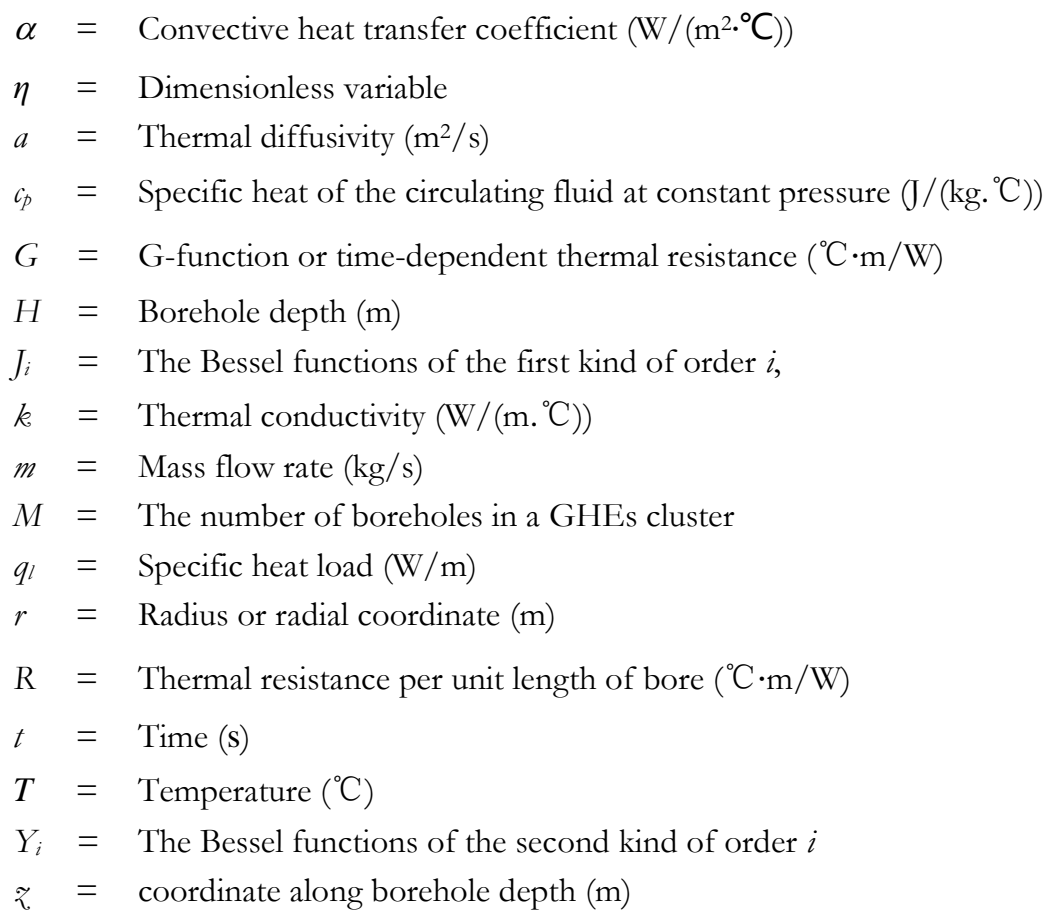

\section{Subscripts}

$$
\begin{aligned}
a & =\text { average } \\
b & =\text { borehole or backfilling material } \\
f & =\text { fluid } \\
i & =\text { inner } \\
\text { in } & =\text { inlet } \\
m & =\text { mid } \\
o & =\text { outer } \\
\text { out } & =\text { outlet } \\
p & =\text { pipe } \\
s & =\text { soil or ground } \\
0 & =\text { initial }
\end{aligned}
$$

\section{REFERENCES}

Carslaw, H.S., and J.C. Jaeger. 1959. Conduction of heat in solids. Oxford: Clarendon Press, 2nd ed.

Claesson, J., and S. Javed. 2011. An analytical method to calculate borehole fluid temperatures for time-scales from minutes to decades. ASHRAE Transactions 117(2): 279-288.

Hellstrom, G. 1991. Ground heat storage - thermal analysis of duct storage systems I. Theory. Lund, Sweden: University of Lund.

Li, M., and A.C.K. Lai. 2012. New temperature response functions (G functions) for pile and borehole ground heat exchangers based on composite-medium line-source theory. Energy 38: 255-263.

Li, M., and A.C.K. Lai. 2013. Analytical Model for short-time responses of borehole ground heat exchangers: Model development and validation. Applied Energy 104: 510-516.

Li M., P. Li, V. Chan, and A.C.K. Lai. 2014. Full-scale temperature response function (G-function) for heat transfer by borehole ground heat exchangers (GHEs) from sub-hour to decades. Applied Energy 136: 197-205. 
Li, M., and A.C.K. Lai. 2015. Review of analytical models for heat transfer by vertical ground heat exchangers (GHEs): A perspective of time and space scales. Applied Energy 151: 178-191.

Li, M., K. Zhu, and Z. Fang. 2016. Analytical methods for thermal analysis of vertical ground heat exchangers. In S.J. Rees Advances in Ground-Source Heat Pump Systems. Duxford, UK: Elsevier/Woodhead Publishing.

Li, M., X.C. Zhuo, and G.S. Huang. 2017. Improvements on the American Society of Heating, Refrigeration, and Air-Conditioning Engineers Handbook equations for sizing borehole ground heat exchangers. Science and Technology for the Built Environment 23(8): $1267-1281$.

Ma, W.W., M. Li, P. Li, and A.C.K. Lai. 2015. New quasi-3D model for heat transfer in U-shaped GHEs (ground heat exchangers): Effective overall thermal resistance. Energy 90: 578 - 587.

Polyanin, A.D. and V.F. Zaitsev. 2003. Handbook of exact solutions for ordinary differential equations. Boca Raton: Chapman \& Hall/CRC, 2nd ed.

Yang, Y., and M. Li. 2014. Short-time performance of composite-medium line-source model for predicting responses of ground heat exchangers with single U-shaped tube. International Journal of Thermal Sciences 82: 130-137.

Zeng, H.Y., N.R. Diao, and Z.H. Fang. 2002. A finite line-source model for boreholes in geothermal heat exchangers. Heat TransferAsian Research 31: 558-567.

Zeng, H.Y., N.R. Diao, and Z.H. Fang. 2003. Heat transfer analysis of boreholes in vertical ground heat exchangers. International Journal of Heat and Mass Transfer 46(23): 4467-4481. 